\title{
Uso de probióticos en el tratamiento y la prevención de diarrea aguda en niños
}

\author{
Probiotics to prevent and treat acute diarrhea in children \\ Probióticos para o tratamento e prevenção da diarréia aguda em criancas
}

$\underline{\text { Karina Machado }^{1}}$

\section{Resumen}

El principio del uso de probióticos proviene de la utilización de productos fermentados, desde tiempos muy antiguos. Se definen como microorganismos vivos, que administrados en cantidades adecuadas, confieren beneficios para la salud.

Entre las utilidades atribuidas a los probióticos, las más extensamente estudiadas han sido los efectos en niños con diarrea aguda. Existe abundante bibliografía sobre la utilización de estos productos en el tratamiento y la prevención de diarrea aguda infecciosa, y prevención de diarrea nosocomial y de diarrea asociada al uso de antibióticos. Los estudios son heterogéneos en su metodología y muestran resultados dispares, incluso contradictorios. En la interpretación de éstos es imprescindible tener en cuenta las cepas de probióticos utilizadas, las dosis administradas y las características de los pacientes en quienes fueron probadas.

Existe evidencia del beneficio de algunas cepas de probióticos en el tratamiento y la prevención de diarrea aguda en niños. Varias guías de práctica clínica los recomiendan. En Uruguay existe poca oferta de estos productos, y la información disponible es escasa, lo cual dificulta su prescripción segura.

Se revisa en este documento la evidencia científica publicada sobre el uso de probióticos en niños con diarrea aguda.

\footnotetext{
Palabras clave: Probióticos

Disentería

Niño

1. Prof. Agda. Clínica Pediátrica "A". Facultad de Medicina. UDELAR. Máster en Nutrición Pediátrica.

Comité de Nutrición SUP.

Clínica Pediátrica "A". Facultad de Medicina. UDELAR

Trabajo inédito

Declaro no tener conflictos de interés

Fecha recibido: 2/11/19

Fecha aprobado: 31/1/2020

doi: 10.31134/AP.91.1.6
}

Summary

Probiotics from fermented products have been used since ancient times. They are living microorganisms, which, at appropriate levels, can provide health benefits. Among the benefits of probiotics, the most broadly studied has been the effect on children with acute diarrhea. There is extensive literature regarding the use of probiotics for the treatment and prevention of acute infectious diarrhea, for the prevention of nosocomial diarrhea and for antibiotic-caused diarrhea. The studies show heterogeneous methodologies and mixed results, even contradictory. For their interpretation, it is essential to take into account the probiotic strains used, the doses administered and the characteristics of the patients tested.

There is evidence of the benefit of some probiotics strains in the treatment and prevention of acute diarrhea in children. Several Clinical Practice Guidelines recommend them. In Uruguay, there is little supply of these products, and the information available is scarce, which makes them difficult to prescribe.

In the present document, we review the existing published scientific evidence regarding the use of probiotics in children with acute diarrhea. 


\section{Resumo}

Os probióticos de produtos fermentados têm sido utilizados desde os tempos antigos. São microrganismos vivos que, em níveis adequados, podem proporcionar benefícios à saúde.

Entre os benefícios dos probióticos, o mais amplamente estudado tem sido o efeito em crianças com diarréia aguda. Existe extensa literatura sobre o uso de probióticos para o tratamento e prevenção de diarreia infecciosa aguda, para prevenção de diarreia intra-hospitalar e diarreia causada por antibióticos. Os estudos mostram metodologias heterogêneas e resultados mistos, até contraditórios. Para sua interpretação, é essencial levar em consideração as cepas probióticas utilizadas, as doses administradas e as características dos pacientes testados.

Há evidências do benefício de algumas cepas de probióticos no tratamento e prevenção de diarreia aguda em crianças. Várias diretrizes de prática clínica as recomendam. No Uruguai, há pouca oferta desses produtos e a informação disponível é escassa, o que dificulta sua prescrição.

No presente documento, revisamos as evidências científicas publicadas sobre o uso de probióticos em crianças com diarreia aguda.

\section{Palavras chave: Probióticos Disentería Criança}

\section{Introducción}

\section{Definición de probióticos}

El término fue introducido por Wener Kollath en 1953, como "sustancias activas, esenciales para el saludable desarrollo de la vida". En 1992, Fuller los definió como "suplemento alimenticio de microorganismos vivos que afecta de manera beneficiosa al huésped, al mejorar su balance microbiano intestinal" (1).

En 2001, la Food and Agriculture Organization de la Organización Mundial de la Salud (FAO-OMS) definió los probióticos como "microorganismos vivos que, cuando son administrados en cantidades adecuadas, confieren beneficios a la salud"(2,3). Esta ha sido la definición más adoptada y aceptada mundialmente.

En 2011, la Organización Mundial de Gastroenterología publicó sus Guías mundiales para el uso de probió- ticos y prebióticos, revisada en 2017. En este documento se define probióticos como "microbios vivos que pueden agregarse a muchos productos, incluyendo alimentos, medicamentos y suplementos dietéticos"(4).

Un consenso internacional, en 2014, recomendó que se utilice el término probiótico solamente en referencia a productos que contengan microorganismos vivos, en recuento adecuado, de cepas definidas, que puedan otorgar algún beneficio para la salud ${ }^{(3)}$. Según este consenso se pueden definir tres categorías de probióticos de acuerdo a los microorganismos involucrados, el beneficio buscado y la evidencia científica disponible: aquellos utilizados en alimentos o suplementos, sin indicaciones médicas específicas; los que se utilizan en alimentos o suplementos, con indicaciones médicas específicas, y los que se emplean como fármacos ${ }^{(2,3)}$.

\section{Beneficios de los probióticos}

Se les han atribuido muchos beneficios y han sido utilizados en el tratamiento de muchas patologías ${ }^{(5)}$. Se realizaron numerosos estudios para evaluar estas acciones, su seguridad y eficacia, aunque han surgido dificultades al extrapolar efectos identificados en el laboratorio a la clínica $^{(6)}$. El empleo de metaanálisis con cepas específicas sobre efectos particulares puede contribuir a definir mejor su $\mathrm{rol}^{(7)}$.

Algunos beneficios de los probióticos son específicos para determinada cepa, y no extrapolables a otros integrantes de la misma especie. Otros efectos pueden ser comunes a la mayoría de las cepas de un gran grupo taxonómico. Un tercer grupo de beneficios son compartidos por la mayoría de las especies descritas ${ }^{(3,6)}$.

\section{Aplicaciones clínicas}

Se ha implicado a los probióticos en el tratamiento y la prevención de numerosas entidades clínicas ${ }^{(4,5)}$. Sus principales indicaciones en pediatría han sido patologías gastrointestinales, en las que las alteraciones de la microbiota pueden participar en la patogenia: diarrea aguda infecciosa, diarrea asociada al uso de antibióticos, enfermedad inflamatoria intestinal, enterocolitis necrotizante, síndrome de intestino irritable, cólicos del lactante, estreñimiento, etc. Han sido utilizados en la prevención y el tratamiento de afecciones de origen inmunológico como dermatitis atópica, alergia alimentaria, etc. Se han investigado sus efectos en otras numerosas afecciones ${ }^{(3,5,8,9)}$.

La bibliografía de los últimos años abunda en estudios científicos realizados acerca de los beneficios de los probióticos. Existe gran diversidad en el diseño de estos estudios, lo que justifica la variabilidad en los resultados de eficacia y seguridad. Para una mejor sistematización se han clasificado los estudios en niveles de 
evidencia, que han sido tomados para elaborar recomendaciones $^{(4,10)}$.

Como muchos beneficios de estos productos son específicos de cepas o grupos, y de las poblaciones de pacientes en las que fueron probados, se recomienda precaución en la extrapolación de los resultados. Los datos agrupados de distintas cepas podrían llevar a falsas conclusiones. Las recomendaciones de empleo de probióticos deben centrarse en hacer corresponder cepas y dosis del producto utilizado a la situación para la que han mostrado beneficio en ensayos clínicos ${ }^{(3,9)}$.

\section{Objetivos}

\section{Objetivo principal}

- Realizar un análisis de la bibliografía publicada sobre el uso de probióticos en niños con diarrea aguda.

\section{Objetivos secundarios}

- Llevar a cabo una revisión histórica del uso de probióticos en humanos y la evolución del conocimiento científico sobre el tema.

- Realizar una breve puesta a punto acerca de la diarrea aguda infantil como problema de salud a nivel nacional y mundial.

- Analizar las preparaciones comerciales de probióticos sugeridos para el tratamiento de diarrea aguda disponibles en Uruguay.

\section{Metodología}

En la elaboración de este documento se llevaron a cabo búsquedas bibliográficas en MEDLINE, LILACS, SciELO y Cochrane Library. Como estrategia de búsqueda se utilizaron términos MeSH (Medical Subject Headings) y sus equivalentes DeCS (Descriptores de Ciencias de la Salud).

Para el objetivo principal la búsqueda bibliográfica se realizó con el descriptor "probióticos", unido al calificador "uso terapéutico", y el descriptor "diarrea infantil", sumado al calificador "prevención y control", articulados por el operador booleano " $y$ " ("Probiotics/therapeutic use" AND "Diarrhea, Infantile/prevention and control").

Para la revisión histórica y la evolución del conocimiento del uso de probióticos se empleó el descriptor "probióticos" con los calificadores "historia" y "aislamiento y purificación" unidos por el operador booleano "o" ("Probiotics/history" OR "Probiotics/isolation and purification").

Para abordar la diarrea aguda infantil como problema de salud se realizó una búsqueda con el descriptor "diarrea infantil" y los calificadores "epidemiología", etiología", "tratamiento" y "prevención y control" unidos por el operador booleano "o" ("Diarrhea, Infantile/epidemiology" OR "Diarrhea, Infantile/etiology" OR "Diarrhea, Infantile/prevention and control" OR "Diarrhea, Infantile/therapy").

Se seleccionaron artículos publicados en los últimos diez años, ensayos clínicos o revisiones, publicados en inglés, portugués o español, en seres humanos.

También se incluyeron algunos artículos referenciados por la bibliografía correspondiente a las búsquedas primarias y guías de práctica clínica nacionales e internacionales.

Se resumieron las recomendaciones actuales para el uso de probióticos en niños con diarrea aguda de diferentes autores. Se utilizó la clasificación en niveles de evidencia de dichas recomendaciones, empleada a nivel internacional ${ }^{(10)}$.

Para el análisis de las preparaciones comerciales de probióticos sugeridos para tratamiento y prevención de diarrea aguda que se comercializan en Uruguay se utilizaron las fichas técnicas disponibles en la última edición de Farmanuario ${ }^{(11)}$.

\section{Revisión histórica del uso de probióticos en seres humanos}

\section{Historia y antecedentes del uso de} probióticos

El término probiótico deriva del latín y significa "para la vida". El principio de su uso proviene de la utilización de productos fermentados con fines nutricionales y terapéuticos. Se cree que se encontraron espontáneamente. Su historia va en paralelo con la evolución de la raza humana y se remonta a tiempos muy antiguos ${ }^{(2,12)}$.

A finales del siglo XIX, Pasteur concluyó que la fermentación láctica se iniciaba por acción de microorga$\operatorname{nismos}^{(2,7)}$. A inicios del siglo XX, Elie Metchnikoff, considerado padre del uso clínico de probióticos, descubrió cualidades beneficiosas para la salud de la fermentación de la leche, sugiriendo que los microorganismos presentes (identificados posteriormente como Lactobacillus) podrían contrarrestar los efectos dañinos del metabolismo gastrointestinal, que contribuiría a la enfermedad. Metchnikoff declaró que "la dependencia de la flora intestinal de los alimentos hace posible adoptar medidas para modificarla, y reemplazar microorganismos dañinos por útiles". Esta hipótesis científica describe de manera clara el concepto de probiótico ${ }^{(1,2)}$. 
Identificación y empleo de diferentes microorganismos con actividad probiótica

En 1899, Henry Tissier aisló Bifidobacterium de las heces de lactantes amamantados, lo identificó como componente predominante de la flora intestinal y recomendó su administración a lactantes con diarrea ${ }^{(7)}$. Desde el inicio de los años 1900 se utilizaron Streptococcus thermophilus y Lactobacillus delbrueckii subespecie bulgaricus en la fermentación de productos lácteos ${ }^{(1)}$. En 1917, el científico alemán Alfred Nissle aisló una cepa no patogénica de Escherichia coli a partir de las heces de un soldado que no enfermó de enterocolitis, durante un brote severo de shigelosis. Se denominó Escherichia coli cepa Nissle-1917 ${ }^{(4)}$. En 1922, se utilizó Lactobacillus acidophilus en 30 pacientes con constipación, diarrea o eccema, con mejoría de las tres condiciones. Diez años más tarde se confirmó el efecto benéfico de esta cepa en pacientes con constipación ${ }^{(2)}$. En 1930, el microbiólogo japonés Minoru Shirota descubrió bacterias de la flora, que, tras su ingestión, sobrevivían al paso intestinal. De ellas aisló y cultivó Lactobacillus casei, cepa Shirota, que fue utilizada en la elaboración de una bebida fermentada, que se comercializa hasta la actualidad como Yakult ${ }^{(7)}$.

En los años siguientes los estudios se centraron en buscar cepas con efecto probiótico y definir sus mecanismos de acción. Se aislaron productos potenciales de diversos orígenes.

La comprensión de la gran cantidad de microorganismos que habita el intestino, sus complejas interacciones y su capacidad de resistencia ante diversas agresiones fue ampliamente estudiada ${ }^{(2)}$. Se comprendió que el rol de los probióticos está muy relacionado con estos microorganismos $^{(4)}$.

Desde finales de los años 90 se utiliza la denominación "microbiota intestinal" y se la reconoce como la población de microorganismos que habita el intestino y participa activamente en sus procesos, sobre todo homeostasis e inmunidad ${ }^{(5)}$. La adopción del término "microbiota intestinal" no significó solo un cambio en la terminología, sino que se comprendió que está formada por un conjunto de microorganismos que incluyen bacterias, virus protozoos, entre otros, que forman parte de esta comunidad y diversidad, y son beneficiosos para la homeostasis y la salud humana ${ }^{(13)}$.

Se han identificado más de 40.000 especies en el colon y se crearon perfiles de los componentes microbiológicos en humanos sanos ${ }^{(2)}$. Estos conocimientos permitieron entender en qué medida la microbiota se altera, a través de numerosos factores, y a través de qué mecanismos los probióticos pueden corregir o restaurar su equilibrio $^{(2)}$.
Los microorganismos con acción probiótica imitan los efectos positivos de la microbiota autóctona, por lo que cualquier componente de ella podría tener tales efectos. Sin embargo, la mayoría de los que hasta ahora han sido utilizados pertenecen a dos grupos: lactobacilos y bifidobacterias.

En la comunidad científica hay acuerdo en cuanto a la nomenclatura que debe utilizarse para nombrar las cepas de probióticos. Deben identificarse según género, especie, subespecie (si corresponde) y una designación alfanumérica que identifique la cepa ${ }^{(4,6)}$. Se recomienda que los probióticos utilizados en la práctica clínica se vinculen a cepas específicas, cuyos beneficios fueron comprobados en estudios clínicos realizados en huma$\operatorname{nos}^{(4)}$.

\section{Diarrea aguda infantil como problema de salud}

\section{Importancia de la diarrea aguda en niños}

\section{como problema de salud}

La enfermedad diarreica aguda (EDA) es una patología frecuente en niños, especialmente en menores de 5 años. Constituye una de las principales causas de morbimortalidad en la infancia a nivel mundial ${ }^{(14,15)}$. En Uruguay ha sido una enfermedad prevalente, hasta hace pocos años se mantenía como causa de mortalidad infantil*.

El mejoramiento de las condiciones sanitarias de las poblaciones ha logrado disminuir su incidencia. La disminución en la mortalidad vinculada a la enfermedad, lograda en las últimas tres décadas, tiene relación con la distribución y uso extendido de las soluciones de rehidratación oral (SRO) y mejores prácticas nutricionales ${ }^{(14,15)}$.

Aproximadamente $10 \%$ de los niños menores de 5 años que cursan una EDA requieren asistencia sanitaria. La enfermedad representa un porcentaje importante de las consultas a servicios de urgencia y de las hospitalizaciones en salas de cuidados moderados, e incluso en salas de terapia intensiva, representando una importante carga asistencial ${ }^{(16)}$. En Uruguay, durante el año 2012, fue responsable del 12,3\% de los ingresos al Centro Hospitalario Pereira Rossell, centro de referencia para la asistencia de niños beneficiarios del subsector público ${ }^{(17)}$.

${ }^{*}$ https://www.gub.uy/ministerio-salud-publica/tramites-y-servicios/servicios/estadisticas-vitales 


\section{Definición de enfermedad diarreica aguda}

Se denomina EDA a un desorden transitorio, autolimitado, de causa infecciosa, caracterizado por aparición repentina de diarrea, vómitos, fiebre y dolor abdominal.

\section{Etiología de la enfermedad diarreica aguda}

Los principales agentes etiológicos son virus y bacterias. En países desarrollados predominan los primeros, mientras que en poblaciones en desarrollo son más frecuentes los agentes bacterianos. Los rotavirus son el principal agente etiológico de EDA en la infancia en el mundo. Son más frecuentes durante los primeros dos años de vida. Pueden asociar formas graves de enfermedad. En países de clima templado estas infecciones predominan en los meses fríos. Los agentes bacterianos más frecuentes son las diferentes cepas de Escherichia coli. Otras etiologías menos prevalentes son especies de Shigella, Salmonella spp y Campylobacter spp ${ }^{(14,18)}$. En niños con diarrea asociada al uso de antimicrobianos el patógeno más frecuente es Clostridium difficile ${ }^{(14,18,19)}$.

\section{Manifestaciones clínicas de la EDA en niños}

La enfermedad se presenta con deposiciones de consistencia disminuida, náuseas, vómitos, fiebre y dolor abdominal. El hallazgo de sangre, moco o pus en las deposiciones sugiere la presencia de gérmenes enteroinvasores. Las pérdidas hidroelectrolíticas que se producen pueden determinar distintos grados de deshidratación. La pérdida de nutrientes y las dietas restringidas pueden causar malnutrición ${ }^{(14,18)}$.

Se denomina diarrea aguda al conjunto de síntomas que presenta una duración variable, entre pocas horas y siete días. La mayoría de los niños se recupera antes del quinto día ${ }^{(15)}$. Diarrea persistente es la que se inicia en forma aguda y dura 14 días o más ${ }^{(14)}$.

Diarrea nosocomial o intrahospitalaria es la que se contrae durante la estadía hospitalaria. Debe considerarse que el niño no está en período de incubación al ingreso, por lo que se cumple con la definición cuando los síntomas comienzan luego de 48 horas del ingreso. Las infecciones nosocomiales impactan negativamente en el paciente y el sistema sanitario, desde muchos aspectos $^{(20,21)}$. El principal agente etiológico de estas infecciones en niños es rotavirus; $C$. difficile ha sido aislado en porcentajes menores, no quedando claro su rol patogénico $^{(19)}$.

\section{Tratamiento de la EDA}

Los pilares del tratamiento recomendado para niños con EDA tienen como objetivo corregir los disturbios hidroelectrolíticos, si están presentes, y prevenir episodios de deshidratación y alteraciones del estado nutri- cional. No existen medidas terapéuticas destinadas al tratamiento etiológico ${ }^{(18)}$. Se cuenta con recomendaciones nacionales e internacionales para niños con EDA que contemplan diferentes situaciones clínicas $^{(14-16,22,23)}$. Desde 1998 se ha recomendado, a nivel internacional, el uso de probióticos para la prevención y el tratamiento de EDA en niños, así como el tratamiento de la diarrea asociada al uso de antibióticos ${ }^{(14,15)}$. En Uruguay, las Normas Nacionales de Atención Pediátrica, en su última edición del año 2014, no incluyen esta recomendación ${ }^{(23)}$. Sin embargo, en su práctica clínica, un porcentaje importante de pediatras indican estos productos a niños con diarrea aguda ${ }^{(24,25)}$.

\section{Probióticos en el tratamiento y la prevención de diarrea aguda en niños}

\section{Introducción}

Desde el inicio de las investigaciones con probióticos en humanos se ha planteado su posible utilidad en el tratamiento y prevención de la diarrea aguda ${ }^{(2,7)}$.

En 1991, E. Isolauri y colaboradores publicaron un artículo sobre un ensayo clínico realizado, en el cual la administración de Lactobacillus casei cepa GG a niños que cursaban una EDA, en su mayoría causada por rotavirus, fue efectiva en acortar el curso de la enfermedad. En 1997, A. Guarino y colaboradores publicaron un artículo sobre una investigación realizada en niños con diarrea leve, en algunos casos causada por rotavirus, a quienes se administró L. casei GG. Los autores concluyeron que la administración del probiótico redujo la duración de la diarrea y la duración de la excreción viral ${ }^{(26)}$.

En los últimos años se ha publicado un muy extenso número de investigaciones, revisiones y metaanálisis sobre el empleo de probióticos en niños con diarrea aguda. Aunque estos estudios tienen metodología y calidad científica diversa, la tendencia actual es afirmar que algunos son beneficiosos en el tratamiento de esta entidad. El grado de evidencia depende de la cepa utilizada y es mayor para los episodios de EDA de etiología vírica ${ }^{(27)}$. Es importante continuar con las investigaciones y nuevos estudios son necesarios, ya que algunos resultados sobre la efectividad de los probióticos en niños con EDA son contradictorios ${ }^{(28)}$.

En la actualidad varias guías de práctica clínica recomiendan el empleo de probióticos en niños sanos, en la prevención y tratamiento de EDA, en la prevención de diarrea asociada a antibióticos y en la prevención de diarrea por C. difficile $e^{(4,8,14,15,29)}$.

Archivos de Pediatría del Uruguay 2020; 91(1) 


\section{Beneficios de los probióticos en el tratamiento de niños con EDA}

Los probióticos tienen diversas acciones en la luz intestinal que podrían ser beneficiosas ante un episodio de EDA. Algunos de estos efectos son compartidos por un gran número de probióticos, mientras que otros son específicos de especie y cepa.

Las acciones generales reconocidas de los probióticos son: efecto antitoxina, actividad antimicrobiana, preservación de las uniones estrechas del epitelio, modulación de la flora intestinal, efecto sobre la actividad metabólica de los enterocitos, y efectos tróficos y antiinflamatorios sobre la mucosa ${ }^{(30,31)}$.

Los principales mecanismos propuestos por los cuales los probióticos reducen la duración de los síntomas en la EDA son: incremento en la producción de ácidos grasos de cadena corta en el colonocito; disminución de la permeabilidad de la barrera intestinal; disminución de la invasión de microorganismos ${ }^{(32)}$. En los casos de EDA y diarrea asociada al uso de antibióticos, los probióticos participan en la reposición de la microbiota, luego de su eliminación. Se propone que estos organismos ocupan la superficie mucosa que quedó deshabita$\mathrm{da}$, atenuando los síntomas y facilitando la recolonización por microorganismos autóctonos ${ }^{(33)}$.

Saccharomyces boulardii induce altos niveles de IgA e interleucina 10 en el intestino, de lo que se puede inducir que participa en la respuesta inmunomoduladora ante la infección ${ }^{(32)}$. Le han sido reconocidos determinados efectos beneficiosos en el tratamiento de niños con EDA, en la prevención de infecciones por $C$. difficile y en la prevención de diarrea asociada al uso de antibióticos: acción antitoxinas, efectos tróficos sobre los enterocitos, efectos antiinflamatorios, estimulación de la respuesta inmune, estimulación de los niveles de disacaridasas, eliminación de toxinas, eliminación de patógenos e interferencia con vías de señalización bacteriana ${ }^{(34)}$.

Lactobacillus rhamnosus cepa GG reduce la acumulación de lípidos y la secreción de sustancias antiinflamatorias, induce la producción de radicales del oxígeno y la producción de sustancias bactericidas, e interfiere con las vías de señalización inducidas por bacterias. Estas acciones serían responsables de sus efectos en la prevención de EDA por rotavirus ${ }^{(34)}$.

Los efectos propuestos para Escherichia coli cepa Nissle-1917 en la prevención de EDA son: interferencia con la motilidad bacteriana y con la permeabilidad de la mucosa a la acción bacteriana ${ }^{(34)}$.

\section{Efectos clínicos de los probióticos en niños con diarrea aguda}

La mayor evidencia sobre la eficacia de los probióticos ha sido descrita en el tratamiento de la diarrea aguda infecciosa. Los mecanismos implicados son: estimulación del sistema inmunitario, competencia por sitios de adherencia en células intestinales y elaboración de sustancias neutralizantes de microorganismos patógenos. Las distintas revisiones sistemáticas realizadas sobre estudios publicados con diferentes cepas concluyen, a pesar de la gran variabilidad de éstos, que los probióticos producen un efecto beneficioso en la evolución de la diarrea aguda infecciosa ${ }^{(3,4,8,9,29)}$.

\section{Tratamiento de la enfermedad diarreica aguda}

Se ha demostrado que el uso de probióticos reduce la duración de la diarrea en casi 25 horas y logra una disminución del número de deposiciones al segundo día de tratamiento ${ }^{(35-40)}$. Los beneficios son más importantes cuanto más precozmente se administre el probiótico. Son más notorios en los episodios causados por rotavirus, aunque también existen beneficios en las infecciones causadas por otros virus. No se ha podido demostrar su eficacia en EDA producida por gérmenes invasores $^{(9,27,30,41)}$. Estos efectos han sido demostrados para $L$. rhamnosus GG, L. reuteri y $S$. boulardii ${ }^{(14,35,42-46)}$.

Estos resultados no han sido demostrados en todas las investigaciones. D. Shnadower y colaboradores publicaron en 2018 un artículo sobre un ensayo clínico controlado realizado en niños con EDA, en el que la administración de L. rhamnosus GG no fue efectiva en disminuir la severidad ni la duración de la enfermedad, ni en prevenir el contagio de los convivientes, frente a placebo $^{(28)}$.

Y. Vandeplas y un grupo de investigadores publicó en 2011 los resultados de su investigación, en la que demostraron un efecto beneficioso de una mezcla de probióticos y prebióticos en niños con diarrea aguda, con disminución en un día en la duración de la enferme$\operatorname{dad}^{(47)}$.

Para otras cepas no se han demostrado estos efectos. S. Freedman y colaboradores en el año 2018 publicaron un estudio realizado con un extenso número de pacientes, en el que no se encontraron diferencias significativas en niños con EDA tratados con L. rhamnosus R0011 y L. helveticus R0052 en cuanto a severidad, duración de la enfermedad ni consultas al departamento de urgen$\operatorname{cias}^{(48)}$. 


\section{Prevención de la enfermedad diarreica aguda}

Hasta el momento son escasos los estudios que concluyen que el uso de probióticos reduce en forma significativa la incidencia de EDA adquirida en la comunidad. $L$. rhamnosus $G G$, L. reuteri y L. casei han mostrado beneficio en la prevención de diarrea por rotavirus en guarderías, con un número necesario para tratar (NNT) de 7, para prevenir un caso ${ }^{(9,49,50)}$.

\section{Prevención de la diarrea asociada al uso de antibióticos}

Se cuenta con evidencia sobre el efecto protector de los probióticos en la prevención de esta entidad ${ }^{(9,39,40)}$. En niños se ha demostrado este efecto para L. rhamnosus GG y $S$. boulardii ${ }^{(30,27)}$. Bifidobacterium lactis y Streptococcus thermophilus han demostrado ser eficaces en reducir la frecuencia de episodios de diarrea asociada a antibióticos en niños ${ }^{(51)}$. L. casei asociado a $B$. breve fueron estudiados en un ensayo clínico randomizado, y no se demostró su efectividad ${ }^{(52)}$.

Una revisión realizada en Colombia, publicada recientemente, concluye que en la diarrea asociada al uso de antibióticos, y específicamente a $C$. difficile, los metaanálisis mostraron una reducción del riesgo entre $50 \%$ y $60 \%$, con una recomendación fuerte para el empleo de L. rhamnosus GG y S. boulardii, con un NNT de 10 (IC95\%: 7-12) $)^{(38)}$.

Diferentes metaanálisis han demostrado que el empleo de probióticos podría ser eficaz en la prevención y el tratamiento de diarrea por C. difficile. Los probióticos que mostraron más eficacia fueron: $S$. boulardii, cepa de $S$. cerevisiae y mezclas de L. acidophilus CL1285 con $L$. casei LBC80R, y L. acidophilus con B. Bifidum ${ }^{(27)}$.

\section{Prevención de la diarrea nosocomial en niños}

En niños hospitalizados, la administración de L. rhamnosus GG, comparada con placebo, puede disminuir la incidencia global de diarrea, incluida la causada por rotavirus ${ }^{(9)}$. La revisión realizada por S. Posadas, publicada recientemente, encontró evidencia de carácter moderado en la prevención de diarrea nosocomial con el uso de L. rhamnosus GG, principalmente de casos por rotavirus. No encontró evidencia suficiente para brindar recomendación sobre $S$. thermophilus y Bifidobacterium bifidum $^{(38)}$.

En 2018 se publicaron las recomendaciones de la European Society for Paediatric Gastroenterology, Hepatology and Nutrition (ESPGHAN) Working Group for Probiotics/Prebiotics sobre la prevención con probióticos de diarrea nosocomial en niños. La conclusión de esta extensa revisión es recomendar la prescripción de L. rhamnosus GG, si se considera el uso de probióticos, con el objetivo de prevenir diarrea nosocomial, con una moderada calidad de la evidencia científica ${ }^{(53)}$.

\section{Recomendaciones actuales para el uso de probióticos en niños con diarrea aguda}

En la tabla 1 se muestran las indicaciones actuales de algunos probióticos en niños con diarrea aguda, según el grado de evidencia científica ${ }^{(4,37,49,50,54,55)}$.

Según la evidencia científica actual, L. rhamnosus $G G, L$. reuteri y $S$. boulardii acortan en un día los episodios de diarrea aguda infecciosa y disminuyen el número de deposiciones luego del segundo día de tratamiento, sobre todo en los causados por rotavirus. L. rhamnosus $G G, L$. reuteri y $L$. casei previenen la diarrea por rotavirus en guarderías, con un NNT de 7. L. rhamnosus $G G$, $S$. boulardii, B. lactis y $S$. thermophilus previenen episodios de diarrea asociada al uso de antibióticos en niños, con un NNT de 10. L. rhamnosus $G G$ previene la diarrea nosocomial en niños, principalmente los casos por rotavirus.

\section{Presentaciones de probióticos disponibles en Uruguay}

En la tabla 2 se muestran las cepas de probióticos recomendadas en niños con diarrea, la dosis y las presentaciones comerciales disponibles en Uruguay ${ }^{(11)}$.

En nuestro país existen pocas presentaciones comerciales que contienen los probióticos cuya efectividad ha sido probada para el tratamiento de niños con diarrea aguda, incluso varios de ellos no se comercializan. Muchos de los probióticos que se encuentran en presentaciones medicamentosas no tienen especificaciones sobre la cepa que contienen, por lo que no se pueden extrapolar los resultados obtenidos en los estudios científicos publicados. En la mayoría de los alimentos/suplementos disponibles en el país, que contienen probióticos, no se especifica la concentración alcanzada del microorganismo en el producto, lo que no permite realizar una recomendación segura. Para poder realizar una prescripción segura de probióticos en niños con diarrea aguda es imprescindible contar con mejor información sobre los productos disponibles en el mercado.

\section{Conclusiones}

- Los beneficios de los probióticos son específicos de las cepas y los pacientes en los que fueron evaluados. Se recomienda precaución en la extrapolación de los resultados. 
Tabla 1. Indicaciones del uso de probióticos en diarrea aguda en niños.

\begin{tabular}{|c|c|c|}
\hline Acción & Cepa de probiótico & $\begin{array}{c}\text { Nivel de evidencia } \\
\text { (The center of evidence) }\end{array}$ \\
\hline \multirow[t]{7}{*}{ Tratamiento de EDA } & L. rhamnosus $G G$ & 1 \\
\hline & S. boulardii & 1 \\
\hline & L. reuteri & 1 \\
\hline & E. coli Nissle-1917 & 3 \\
\hline & L. acidophilus & 3 \\
\hline & L. acidophilus rhamnosus cepas $573 \mathrm{~L} / 1,573 \mathrm{~L} / 2,573 \mathrm{~L} / 3$ & $2^{*}$ \\
\hline & B. clausis cepa UBBC-07 & 2 \\
\hline \multirow[t]{7}{*}{ Prevención de EDA } & L. rhamnosus $G G$ & 2 \\
\hline & S. boulardii & 2 \\
\hline & L. reuteri cepa DSM 17938 & 2 \\
\hline & L. acidophilus & 2 \\
\hline & L. casei Shirota & 2 \\
\hline & L. bulgaricus & 2 \\
\hline & B. lactis & 2 \\
\hline \multirow{2}{*}{$\begin{array}{l}\text { Prevención de diarrea asociada a } \\
\text { antibióticos }\end{array}$} & L. rhamnosus $G G$ & 1 \\
\hline & S. boulardii & 1 \\
\hline \multicolumn{3}{|l|}{ Prevención de diarrea nosocomial } \\
\hline & L. rhamnosus $G G$ & 1 \\
\hline & S. thermophiles & 2 \\
\hline & B. lactis cepa $\mathrm{Bb} 12$ & 2 \\
\hline & B. bifidum & 2 \\
\hline \multicolumn{3}{|c|}{ Niveles de evidencia (The center of evidence) } \\
\hline 1 & Revisión sistemática de ensayos clínicos randomizados. & \\
\hline 2 & Ensayo clínico randomizado individual. & \\
\hline 3 & Revisión sistemática de estudios de cohorte. & \\
\hline 4 & $\begin{array}{l}\text { Serie de casos, estudios de control de casos, o estudios } \\
\text { controlados históricamente. }\end{array}$ & \\
\hline 5 & Razonamiento basado en un mecanismo. & \\
\hline
\end{tabular}


Tabla 2. Cepas de probióticos recomendadas en niños con diarrea, dosis y presentaciones comerciales disponibles en Uruguay.

\begin{tabular}{|c|c|c|c|}
\hline Cepa de probiótico & Dosis recomendada & Presentaciones medicamentosas & $\begin{array}{l}\text { Presentaciones como } \\
\text { alimento/suplemento }\end{array}$ \\
\hline L. rhamnosus $G G$ & $\begin{array}{l}1-2 \geq 10^{10} \text { UFC/día } \\
\text { Tratamiento EDA: } 5-7 \text { días }\end{array}$ & & Yogur Vital + (Conaprole) $)^{* *}$ \\
\hline S. boulardii cepa CNCM I-745 & $\begin{array}{l}250-750 \mathrm{mg} / \text { día } \\
\text { Tratamiento EDA: } 5-7 \text { días }\end{array}$ & $\begin{array}{l}\text { Deoflora }{ }^{\circledR}(1 \text { cápsula o } \\
1 \text { sobre }=200 \mathrm{mg})^{*}\end{array}$ & \\
\hline L. reuteri cepa DSM 17938 & $\begin{array}{l}10^{8} \text { a } 4 \times 10^{8} \mathrm{UFC} / \text { día } \\
\text { Tratamiento EDA: } 5-7 \text { días }\end{array}$ & Glutapak $\mathrm{R}^{\circledR}\left(1 \text { sobre }=10^{8} \text { UFC }\right)^{*}$ & \\
\hline L. acidophilus & $\begin{array}{l}10^{10} \text { UFC/día } \\
\text { Tratamiento de EDA: } 5-9 \text { días }\end{array}$ & $\begin{array}{l}\text { Biotica Pro }^{\circledR}(1 \text { cápsula }= \\
5 \times 10^{11} \text { UFC }^{-} \\
\text {Biotica Pro Kid }{ }^{\circledR}(1 \text { medida }= \\
\left.1,9 \times 10^{8} \text { UFC }\right) \\
\text { Biotica Pro Cran }{ }^{\circledR}(1 \text { cápsula }= \\
\left.7 \times 10^{8} \text { UFC }\right) \\
\text { Multiflora }^{\circledR}\left(1 \text { medida }=10^{9} \text { UFC }\right)\end{array}$ & Yogur Bio Transit (Conaprole) ${ }^{* *}$ \\
\hline B. clausis cepa UBBC-07 & $\begin{array}{l}2 \times 10^{9} \text { UFC dos veces al día } \\
\text { Tratamiento EDA: } 10 \text { días }\end{array}$ & $\begin{array}{l}\text { Enterogermina }^{\circledR}(5 \mathrm{ml}= \\
\left.2 \times 10^{9} \mathrm{UFC}\right)^{*} \\
\text { Enterogermina plus }^{\circledR}(5 \mathrm{ml}= \\
\left.4 \times 10^{9} \mathrm{UFC}\right)^{*}\end{array}$ & \\
\hline L. casei cepa Shirota & $10^{10}$ UFC/día & 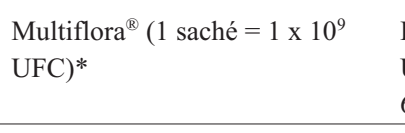 & $\begin{array}{l}\text { Leche fermentada Yacult (Yacult } \\
\text { Uruguay). Frasco } 80 \mathrm{~g}= \\
6,5 \times 10^{9} \text { UFC }\end{array}$ \\
\hline L. bulgaricus & $10^{9} \mathrm{UFC} /$ día & $\begin{array}{l}\text { Biotica Pro }^{\circledR}(1 \text { cápsula }= \\
\left.5 \times 10^{11} \text { UFC }\right) \\
\text { Multiflora }^{\circledR}\left(1 \text { medida }=10^{9} \text { UFC }\right)\end{array}$ & \\
\hline B. lactis cepa $\mathrm{Bb} 12$ & $\begin{array}{l}\text { 14,5 x } 10^{6} \text { UFC/día } \\
\text { Tratamiento EDA: } 7 \text { días }\end{array}$ & & Yogur Bio Transit (Conaprole)** \\
\hline S. thermophilus & $10^{9} \mathrm{UFC} /$ día & $\begin{array}{l}\text { Biotica Pro }{ }^{\circledR}(1 \text { cápsula }= \\
\left.5 \times 10^{11} \text { UFC }\right) \\
\text { Biotica Pro Kid }{ }^{\circledR}(1 \text { medida }= \\
\left.1,35 \times 10^{8} \text { UFC }\right) \\
\text { Biotica Pro Cran }{ }^{\circledR}(1 \text { cápsula }= \\
\left.7 \times 10^{8} \text { UFC }\right) \\
\text { Multiflora }^{\circledR}\left(1 \text { medida }=10^{9} \text { UFC }\right)\end{array}$ & \\
\hline B. bifidum & $\begin{array}{l}3 \times 10^{9} \text { UFC/día } \\
\text { Tratamiento de EDA: } 5 \text { días }\end{array}$ & $\begin{array}{l}\text { Biotica Pro }{ }^{\circledR}(1 \text { cápsula }= \\
\left.5 \times 10^{11} \text { UFC }\right) \\
\text { Biotica Pro Kid }{ }^{\circledR}(1 \text { medida }= \\
\left.2,6 \times 10^{8} \text { UFC }\right) \\
\text { Biotica Pro Cran }^{\circledR}(1 \text { cápsula }= \\
\left.7 \times 10^{8} \text { UFC }\right)\end{array}$ & \\
\hline \multicolumn{4}{|c|}{$\begin{array}{l}\text { Glutapak } \mathrm{R}^{\circledast} \text { contiene también prebióticos. } \\
\text { Biotica Pro }{ }^{\circledast} \text { contiene prebióticos y mezcla de probióticos ( } L \text {. acidophil } \\
\text { Biotica Pro Kid }{ }^{\circledR} \text { contiene prebióticos y mezcla de probióticos }(L \text {. acido } \\
\text { mophilus y L. casei). } \\
\text { Biotica Pro Cran }{ }^{\circledast} \text { contiene extracto de cranberry, vitamina C y mezcla } \\
\text { Multiflora }^{\circledR} \text { contiene prebióticos y mezcla de probióticos }(L \text {. casei, } L \text {. rh } \\
\text { bulgaricus). No se especifica cantidad de UFC de cada probiótico que c } \\
\text { * En la bibliografía no se especifica qué cepa contiene. } \\
\text { ** En la bibliografía no se especifica la cantidad de UFC por porción. } \\
\text { UFC: unidades formadoras de colonias. }\end{array}$} \\
\hline
\end{tabular}


- Desde hace más de 20 años se recomienda el uso de probióticos en el tratamiento y la prevención de la diarrea aguda.

- Según la evidencia científica actual pocas cepas han demostrado su utilidad en el tratamiento y la prevención de la enfermedad, con resultados discretos.

- En Uruguay existen pocas presentaciones comerciales con las cepas de probióticos cuya efectividad ha sido probada en el tratamiento de niños con diarrea aguda. Muchos de estos productos no cuentan con la información necesaria para realizar una prescripción segura.

\section{Referencias bibliográficas}

1. Gasbarrini G, Bonvicini F, Gramenzi A. Probiotics History. J Clin Gastroenterol 2016; 50(Suppl 2): S116-9.

2. McFarland L. From yaks to yogurt: the history, development, and current use of probiotics. Clin Infect Dis 2015; 60(Suppl 2):S85-90.

3. Hill C, Guarner F, Reid G, Gibson G, Merenstein D, Pot B, et al. Expert consensus document. The International Scientific Association for Probiotics and Prebiotics consensus statement on the scope and appropriate use of the term probiotic. Nat Rev Gastroenterol Hepatol 2014; 11(8):506-14

4. World Gastroenterology Organization. Guías mundiales de la Organización Mundial de Gastroenterología: probióticos y prebióticos. Milwaukee, WI: WGO, 2017. Disponible en: https:/www.worldgastroenterology.org/guidelines/global-guidelines/probiotics-and-prebiotics/probiotics-and-prebiotics-spanish. [Consulta 21 setiembre 2019].

5. Castañeda C. Probióticos, puesta al día. Rev Cubana Pediatr 2018; 90(2):286-98

6. Sanders ME, Merenstein D, Merrifield C, Hutkins R. Probiotics for human use. Nutr Bull 2018; 43(3):212-25.

7. Gogineni V, Morrow L, Gregory P, Malesker M. Probiotics: history and evolution. J Anc Dis Prev Rem 2013; 1:107.

8. Cruchet S, Furnes R, Maruy A, Hebel E, Palacios J, Medina $\mathbf{F}$, et al. The use of probiotics in pediatric gastroenterology: a review of the literature and recommendations by Latin-American experts. Paediatr Drugs 2015; 17(3):199-216.

9. Román E, Álvarez G. Empleo de probióticos y prebióticos en pediatría. Nutr Hosp 2013; 28(Supl 1):42-5.

10. The Center for Evidence-Based Medicine. CEBM [web page]. Oxford, UK: University of Oxford, 2019. Disponible en: https://www.cebm.net. [Consulta: 21 setiembre 2019].

11. González L, de Oliveira R. Farmanuario Uruguay: guía farmacológica y terapéutica. 29 ed. Montevideo: Informédica, 2019 .

12. Ozen M, Dinleyici E. The history of probiotics: the untold story. Benef Microbes 2015; 6(2):159-65.

13. Icaza M. Microbiota intestinal en la salud y la enfermedad. Rev Gastroenterol Méx 2013; 78(4):240-8.

14. Guarino A, Albano F, Ashkenazi S. European Society for Paediatric Gastroenterology, Hepatology, and Nutrition/Eu- ropean Society for Paediatric Infectious Diseases Evidence-based Guidelines for the Management of Acute Gastroenteritis in Children in Europe. J Pediatr Gastroenterol Nutr 2008; 46(Suppl 2):S81-122.

15. Gutiérrez P, Salazar E, Polanco I. Guía de práctica clínica ibero-latinoamericana sobre el manejo de la gastroenteritis aguda en menores de 5 años: enfoque, alcances y diseño. An Pediatr (Barc) 2014; 80(Supl 1): 1-4.

16. National Institute for Health and Clinical Excellence. Diarrhoea and vomiting caused by gastroenteritis in under $5 \mathrm{~s}$ : diagnosis and management. Clinical guideline [CG84]. London: NICE, 2009. Disponible en: https://www.nice.org. uk/guidance/cg84. [Consulta 21 setiembre 2019].

17. Notejane M, Pandolfo S, García L, Parada M, Coedo V, Betancor L, et al. Gastroenteritis aguda: formas de presentación clínica y etiología en niños hospitalizados en el Hospital Pediátrico, Centro Hospitalario Pereira Rossell, año 2012. Arch Pediatr Urug 2015; 86(2):91-7.

18. Román E, Barrio J, López MJ. Diarrea aguda. En: Asociación Española de Pediatría, Sociedad Española de Gastroenterología, Hepatología y Nutrición Pediátrica. Protocolos diagnóstico-terapéuticos de gastroenterología, hepatología y nutrición pediátrica. Madrid: Ergon, 2010:11-20.

19. Rodríguez P, Cofré J. Diarrea asociada a Clostridium difficile en niños. Rev Chil Infect 2015; 32(5):550-8.

20. World Health Organization. Prevention of hospital-acquired infections: a pratical guide. Geneva: WHO, 2002.

21. World Health Organization. Patient Safety. Report on the Burden of Endemic Health Care-associated Infection Worldwide: clean care is safer care. Geneva: WHO, 2011.

22. World Health Organization. The treatment of diarrhea: a manual for physicians and other senior health workers. Ginebra: WHO, 2005. Disponible en: https://apps.who.int/ iris/bitstream/handle/10665/43209/9241593180.pdf?sequen$\mathrm{ce}=1$. [Consulta 21 setiembre 2019].

23. Giachetto G, Pérez W, Pírez C, Prego J. Atención pediátrica: normas nacionales de diagnóstico, tratamiento y prevención. 8 ed. Montevideo: Oficina del Libro-FEFMUR, 2014.

24. Vázquez M, Iglesias S, Iglesias C, Pérez W. Utilización de probióticos, antisecretores y zinc en la gastroenteritis aguda en pediatría en Uruguay. Arch Pediatr Urug 2019; 90(3):126-37.

25. Isolauri E, Juntunen M, Rautanen T, Sillanaukee P, Koivula T. A human Lactobacillus strain (Lactobacillus casei $\mathrm{sp}$ strain GG) promotes recovery from acute diarrhea in children. Pediatrics 1991; 88(1):90-7.

26. Guarino A, Canani R, Spagnuolo M, Albano F, Di Benedetto $\mathbf{L}$. Oral bacterial therapy reduces the duration of symptoms and of viral excretion in children with mild diarrhea. J Pediatr Gastroenterol Nutr 1997; 25(5):516-9.

27. Díaz J, González D. Aplicaciones en gastroenterología. Nutr Hosp 2015; 31(Supl. 1):21-5.

28. Schnadower D, Tarr P, Casper T, Gorelick M, Dean J, O'Connell K, et al. Lactobacillus rhamnosus GG versus Placebo for Acute Gastroenteritis in Children. N Engl J Med 2018; 379(21):2002-14

29. Szajewska H, Guarino A, Hojsak I, Indrio F, Kolacek S, Shamir R, et al. Use of probiotics for management of acute gastroenteritis: a position paper by the ESPGHAN Working 
Group for Probiotics and Prebiotics. J Pediatr Gastroenterol Nutr 2014; 58(4):531-9.

30. Álvarez G, Pérez J, Tolín M, Sánchez C. Recomendaciones para el empleo de probióticos en la diarrea en la infancia. Acta Pediatr Esp 2017; 75(5-6):56-60.

31. Thomas D, Greer F. Probiotics and prebiotics in pediatrics. Pediatrics 2010; 126(6):1217-31.

32. Vieira A, Teixeira M, Martins F. The role of probiotics and prebiotics in inducing gut immunity. Front Immunol 2013; $4: 445$.

33. Suárez J. Microbiota autóctona, probióticos y probióticos. Nut Hosp 2013; 28(Supl 1):38-41.

34. Pothoulakis C. Review article: anti-inflammatory mechanisms of action of Saccharomyces boulardii. Aliment Pharmacol Ther 2009; 30(8):826-33.

35. Allen S, Martinez E, Gregorio G, Dans L. Probiotics for treating acute infectious diarrhoea. Cochrane Database Syst Rev 2010; 2010(11):CD003048.

36. Applegate J, Fischer Walker C, Ambikapathi R, Black R. Systematic review of probiotics for the treatment of community-acquired acute diarrhea in children. BMC Public Health 2013; 13(Suppl 3):S16.

37. Ahmadi E, Alizadeh R, Rezai M. Efficacy of probiotic use in acute rotavirus diarrhea in children: A systematic review and meta-analysis. Caspian J Intern Med 2015; 6(4):187-95.

38. Posada S, Vera J. Probióticos en diarrea aguda, asociada a antibióticos y nosocomial: evidencia en pediatría. Rev Colomb Gastroenterol 2018; 33(1):41-8.

39. Pérez C. Probióticos en la diarrea aguda y asociada al uso de antibióticos en pediatría. Nutr Hosp 2015; 31(Supl 1):64-7.

40. Hojsak I. Probiotics in Children: What Is the Evidence? Pediatr Gastroenterol Hepatol Nutr 2017; 20(3):139-46.

41. Erdoðan O, Tanyeri B, Torun E, Gönüllüi E, Arslan H, Erenberk U, et al. The comparition of the efficacy of two different probiotics in rotavirus gastroenteritis in children. $\mathbf{J}$ Trop Med 2012; 2012:787240.

42. Szajewska H, Skórka A. Saccharomyces boulardii for treating acute gastroenteritis in children: updated meta-analysis of randomized controlled trials. Aliment Pharmacol Ther 2009; 30(9):960-1.

43. Corrêa N, Penna F, Lima F, Nicoli J, Filho L. Treatment of acute diarrhea with Saccharomyces boulardii in infants. J Pediatr Gastroenterol Nutr 2011; 53(5):497-501.

44. Szajewska H, Skórka A, Ruszczyñski M, Gieruszczak D. Meta-analysis: Lactobacillus GG for treating acute gastroenteritis in children - updated analysis of randomised controlled trials. Aliment Pharmacol Ther 2013; 38(5):467-76.
45. Feizizadeh S, Salehi A, Akbari V. Efficacy and safety of Saccharomyces boulardii for acute diarrhea. Pediatrics 2014; 134(1):e176-91.

46. Kelesidis T, Pothoulakis C. Efficacy and safety of the probiotic Saccharomyces boulardii for the prevention and therapy of gastrointestinal disorders. Therap Adv Gastroenterol 2012; 5(2):111-25.

47. Vandenplas Y, De Hert S. Randomised clinical trial: the synbiotic food supplement Probiotical vs. placebo for acute gastroenteritis in children. Aliment Pharmacol Ther 2011; 34(8):862-7.

48. Freedman S, Williamson S, Farion K, Gouin S, Willan A, Poonai N, et al. Multicenter Trial of a Combination Probiotic for Children with Gastroenteritis. N Engl J Med 2018; 379(21):2015-26.

49. Sazawal S, Hiremath G, Dhingra U, Malik P, Deb S, Black R. Efficacy of probiotics in prevention of acute diarrhoea: a meta-analysis of masked, randomised, placebo-controlled trials. Lancet Infect Dis 2006; 6(6):374-82.

50. Guandalini S. Probiotics for prevention and treatment of diarrhea. J Clin Gastroenterol 2011; 45(Suppl):S149-53.

51. Corrêa N, Péret L, Penna F, Lima F, Nicoli J. A randomized formula controlled trial of Bifidobacterium lactis and Streptococcus thermophilus for prevention of antibiotic-associated diarrhea in infants. J Clin Gastroenterol 2005; 39(5): 385-9.

52. Souza D, Tanús J. Efeito de Lactobacillus casei e Bifidobacterium breve na diarréia associada ao uso de antimicrobiano: estudo clínico randomizado duplo cego. Rev Soc Bras Med Trop 2012; 45 (1):112-6.

53. Hojsak I, Szajewska H, Canani R, Guarino A, Indrio F, Kolacek S, et al. Probiotics for the Prevention of Nosocomial Diarrhea in Children. J Pediatr Gastroenterol Nutr 2018; 66(1):3-9.

54. Urbañska M, Gieruszczak D, Szajewska H. Systematic review with meta-analysis: Lactobacillus reuteri DSM 17938 for diarrhoeal diseases in children. Aliment Pharmacol Ther 2016; 43(10):1025-34.

55. Sudha M, Jayanthi N, Pandey D, Verma A. Bacillus clausii UBBC-07 reduces severity of diarrhoea in children under 5 years of age: a double blind placebo controlled study. Benef Microbes 2019; 10(2):149-154.

Correspondencia: Dra. Karina Machado.

Correo electrónico: kmachado30@gmail.com 